\title{
Digital Subtraction Angiography Findings in Aneurysmal Subarachnoid Haemorrhage: Experience of 30 Cases in Bangladesh
}

\author{
Mahmudul Islam¹, Mashfiqul Hasan², Maliha Hakim³, Sharif Uddin Khan, \\ Kazi Mohibur Rahman', Quazi Deen Mohammad ${ }^{6}$
}

\begin{abstract}
${ }^{1}$ Assistant Professor, Department of Neurology, National Institute of Neurosciences and Hospital, Dhaka, Bangladesh;
${ }^{2}$ Assistant Registrar, Department of Neurology, National Institute of Neurosciences and Hospital, Dhaka, Bangladesh;

${ }^{3}$ Professor, Department of Neurology, National Institute of Neurosciences and Hospital, Dhaka, Bangladesh;

${ }^{4}$ Associate Professor, Department of Neurology, National Institute of Neurosciences and Hospital,

Dhaka, Bangladesh; ${ }^{5}$ Associate Professor, Department of Intervention Neurology, National

Institute of Neurosciences and Hospital, Dhaka, Bangladesh; ${ }^{6}$ Director \& Professor of

Neurology, National Institute of Neurosciences and Hospital, Dhaka, Bangladesh
\end{abstract}

[Received: 21 January 2016; Revised: 6 March 2016; Accepted: 11 June 2016; Published: 1 July 2016]

\begin{abstract}
Background: Digital subtraction angiography (DSA) is an important diagnostic tool for aneurysmal subarachnoid haemorrahage (SAH). Objectives: The purpose of the present study was to observe the findings of DSA in patients with SAH. Methodology: This cross-sectional study was performed in the Department of Neurology at Dhaka Medical College Hospital, Dhaka from December 2009 to November 2010 for a period of one (1) year. Patients at any age in both sexes who were admitted with SAH were included by purposive sampling technique. Demographic and clinical data were recorded in a semi-structured questionnaire. Angiography was performed after 4 weeks by DSA technique among all patients. Results: The mean age of the participants was $44.9 \pm 9.7$ years $(m e a n \pm S D)$. Male female ratio was 3:2. Hypertension, smoking, diabetes mellitus and family history of SAH were found in $14(46.6 \%)$ cases, $13(43.3 \%)$ cases, $3(10.0 \%)$ cases and $2(6.7 \%)$ cases patients respectively. All the study patients $(100 \%)$ had headache and vomiting while $19(63.3 \%)$ cases had history of unconsciousness. Signs of meningeal irritation were present in $23(76.7 \%)$ patients. Anterior communicating artery was the most common site of aneurysm (36.7\%) followed by middle cerebral $(26.7 \%)$ and posterior communicating artery $(23.3 \%)$. Majority of aneurysms were medium sized (46.7\%), while $33.3 \%$ were small and $20.0 \%$ were large. Saccular aneurysm was found in $93.3 \%$ and aneurysms of narrow neck size in $86.7 \%$. Conclusions: DSA has demonstrated that anterior communicating artery is the most common site of aneurysms in patients with SAH and is most often saccular with a narrow neck. [Journal of National Institute of Neurosciences Bangladesh, 2016;2(2): 51-54]
\end{abstract}

Keywords: Digital subtraction angiography; subarachnoid haemorrhage; aneurysmal

Correspondence: Dr. Mahmudul Islam, Assistant Professor, Department of Neurology, National Institute of Neurosciences and Hospital, Sher-E-Bangla Nagar, Agargaon, Dhaka-1207, Bangladesh; Email: drmahmudul@yahoo.com; Cell no: +8801822496267 Conflict of interest: There is no conflict of interest relevant to this paper to disclose.

Funding agency: This research project was not funded by any group or any institution.

Contribution to authors: MI, SUK \& KMR were involved in protocol preparation, data collection and literature search up to manuscript writing. MH \& QDM were involved in preparation and revision of this manuscript.

How to cite this article: Islam M, Hasan M, Hakim M, Khan SU, Rahman KM, Mohammad QD. Digital Subtraction Angiography Findings in Aneurysmal Subarachnoid Haemorrhage: Experience of 30 Cases in Bangladesh. J Natl Inst Neurosci Bangladesh, 2016;2(2): 51-54

Copyright: (C2016 Islam et al. Published by Journal of National Institute of Neurosciences Bangladesh. This article is published under the Creative Commons CC BY-NC License (https://creativecommons.org/licenses/by-nc/4.0/). This license permits use, distribution and reproduction in any medium, provided the original work is properly cited, and is not used for commercial purposes.

\section{Introduction}

Although aneurysmal subarachnoid haemorrhage (SAH), accounts for only $3 \%$ of all strokes ${ }^{1}$, it is responsible for $5 \%$ of stroke deaths and for more than one-quarter of potential life-years lost through stroke ${ }^{2}$. In the 20th century, there had been great advances in diagnosis and treatment of SAH. However, there is only modest improvement in overall outcome ${ }^{3}$. Hence, there 
are still formidable challenges ahead for neurologists, neurosurgeons and radiologists. It is well recognized that, after initial stabilization, patients with SAH should be transferred to centers with a dedicated neurological critical care unit where one of the main goals of treatment is the prevention of rebleeding ${ }^{4}$. After detection of the aneurysms, it is suggested to secure them as early as possible to prevent rebleeding, which is done either by operative clipping or by endovascular coiling. Proper localization and actual sizing of the aneurysms are obligatory before such procedure. This can be done accurately by digital subtraction angiography (DSA) .

Prior to the advent of newer techniques, conventional angiography was the test of choice for most neck vessel and cerebral pathology. The basic procedure was to inject contrast media into the arterial system rendering this normally radiolucent structure radio-opaque. Two-dimensional (2D) film was then developed yielding resolution comparable to plain radiographs ${ }^{6}$. The digital subtraction techniques were accomplished by associated computer technology, resulting in greater contrast between transient blood flow within the vasculature and permanent structures (bones). Later developments in robotic $\mathrm{C}$-arms allowed larger image intensifiers to be manipulated in multiple plains creating multiple views with image resolution greater than conventional angiography. The multiple views obtained have also been manipulated into three-dimensional (3D) images and movies allowing further analysis of cerebral aneurysms regarding their location, size and morphology. The introduction of nonionic contrast agents further increased image quality and reduced patient's risk of complication. With these advancements DSA is now considered as the gold standard in aneurysmal $\mathrm{SAH}^{7}$. In this background, the current study was undertaken to observe the clinical and demographic profile and the findings of DSA in a tertiary hospital of Bangladesh.

\section{Methodology}

This cross-sectional study was performed in the Department of Neurology at Dhaka Medical College Hospital, Dhaka from December 2009 to November 2010 for a period of one (1) year. Patients at any age in both sexes who were admitted with SAH were included by purposive sampling technique. Written informed consent was taken from the participants. The project was run after approval of the local ethical board. The SAH was diagnosed on the basis of clinical feature and findings of CT scan of brain. Those having traumatic $\mathrm{SAH}$ or Intra-cerebral haemorrhage were excluded from the study. Demographic and clinical data were recorded by a semi-structured questionnaire. Angiography was performed after 4 weeks by DSA technique via a femoral arterial approach by interventional neurologists. Identification of aneurysmal sites and measurement of their size were done. Aneurysmal size $\leq 4 \mathrm{~mm}$ was considered as small, $5-12 \mathrm{~mm}$ as medium and $\geq 13 \mathrm{~mm}$ as large ${ }^{8}$. Morphology of the aneurysms along with size of the neck and neck body ratio was assessed. Data were expressed as frequencies or percentages and mean $( \pm \mathrm{SD})$ as applicable.

\section{Results}

The mean age of the participants was $44.9 \pm 9.7$ years (mean \pm SD), ranging from 26 to 70 . Most of the patients were in the age group of $41-50$ years $(53.3 \%)$. Male female ratio was $3: 2(60 \%$ male, $18 / 30 ; 40 \%$ female, 12/30).Hypertension, smoking, diabetes mellitus and family history of SAH were found in 14 (46.6\%), $13(43.3 \%), 3(10.0 \%)$ and $2(6.7 \%)$ patients respectively (Table 1).

Table 1: Baseline Characteristics of the Respondents $(\mathrm{n}=30)$

\begin{tabular}{lc}
\hline Characteristics & $\mathbf{n}(\mathbf{\%})$ \\
\hline Age (years, mean \pm SD) & $44.9 \pm 9.7$ \\
Age groups & \\
• $\leq 30$ years & $2(6.7 \%)$ \\
- $31-40$ & $6(20.0 \%)$ \\
- $41-50$ & $16(53.3 \%)$ \\
• $\geq 51$ & $6(20.0 \%)$ \\
Sex & \\
• Male & $18(60.0 \%)$ \\
- Female & $12(40.0 \%)$ \\
Risk factors & \\
• Hypertension & $14(46.6 \%)$ \\
• Diabetes mellitus & $3(10.0 \%)$ \\
- Smoking & $13(43.3 \%)$ \\
• Family history of SAH & $2(6.7 \%)$ \\
\hline
\end{tabular}

SAH: subarachnoid hemorrhage

Regarding the clinical presentation, it was observed that all the study patients $(100 \%)$ had headache and vomiting, while 19 (63.3\%) were found unconscious and $3(10 \%)$ experienced double vision. Weakness of one side,blurring of vision and vertigo was present in 1 (3.3\%) patient each. Signs of meningeal irritation were present in $23(76.7 \%)$ patients. Third cranial nerve palsy was found in $3(10 \%)$ and hemiplegia in $1(3.3 \%)$ of the study patients (Table 2). 
Table 2: Presenting Features of the Respondents $(n=30)$

\begin{tabular}{lc}
\hline Characteristics & n (\%) \\
\hline Symptoms & \\
- Headache & $30(100.0 \%)$ \\
- Vomiting & $30(100.0 \%)$ \\
- Unconsciousness & $19(63.3 \%)$ \\
- Double vision & $3(10.0 \%)$ \\
- Weakness of one side & $1(3.3 \%)$ \\
- Blurring of vision & $1(3.3 \%)$ \\
- Vertigo & $1(3.3 \%)$ \\
Signs of meningeal irritation & \\
- Present & $23(76.6 \%)$ \\
- Absent & $7(23.3 \%)$ \\
Focal neurological deficit & \\
- Third cranial nerve palsy & $3(10.0 \%)$ \\
- Hemiplegia & $1(3.3 \%)$ \\
\hline
\end{tabular}

DSA identified aneurysm in all the participants. It was observed that anterior communicating artery was the most common site of aneurysm (36.7\%), followed by middle cerebral $(26.7 \%)$ and posterior communicating artery $(23.3 \%)$. Other sites include postero-inferior cerebellar artery $(6.7 \%)$, internal carotid artery $(3.3 \%)$ and basilar artery $(3.3 \%)$. Majority of aneurysms were medium sized $(46.7 \%)$, while $33.3 \%$ were small and $20.0 \%$ were large. Regarding the morphology, saccular aneurysm was found in $28(93.3 \%)$ and non-saccular in only $2(6.7 \%)$ study patients.Most of the study patients $(86.7 \%)$ had aneurysms of narrow neck size and rest $(13.3 \%)$ had wide neck aneurysm (Table 3 ).

Table 3: Presenting Features of the Respondents $(n=30)$

\begin{tabular}{lc}
\hline Characteristics & n (\%) \\
\hline Location of aneurysm & \\
- Internal carotid artery & $1(3.3 \%)$ \\
- Anterior communicating artery & $11(36.7 \%)$ \\
- Middle cerebral artery & $8(26.7 \%)$ \\
- Posterior communicating artery & $7(23.3 \%)$ \\
- Postero-inferior cerebellar artery & $2(6.7 \%)$ \\
- Top of the basilar artery & $1(3.3 \%)$ \\
Size of aneurysm & \\
- Small ( $\leq 4$ mm) & $10(33.3 \%)$ \\
- Medium (5-12 mm) & $14(46.7 \%)$ \\
- Large ( $\geq 13$ mm) & $6(20.0 \%)$ \\
Morphology of aneurysm & \\
- Saccular & $28(93.3 \%)$ \\
- Non- saccular & $2(6.7 \%)$ \\
Neck size of aneurysm & \\
- Narrow & $26(86.7 \%)$ \\
- Wide & $4(13.3 \%)$ \\
\hline
\end{tabular}

DSA: Digital subtraction angiography

\section{Discussion}

This observational study was carried to evaluate the findings of DSA in SAH in a tertiary hospital of Bangladesh. It was observed that the procedure was successful to provide important information that was necessary to plan subsequent procedure to prevent rebleed from the aneurysm. The study also represented the clinical and demographic profile of the patients of $\mathrm{SAH}$ and the pattern of aneurysm in respect to location, size and morphology.

In the present study, more than half $(53.3 \%)$ of the patients were in the age group of 41 to 50 years and the mean age was also around 45 years which closely agrees with the studies done by other authors ${ }^{9-10}$. In few studies, mean age of the SAH was found higher ${ }^{5,11-13}$. The higher age of presentation may be related to longer life expectancy in their study populations. Though the male and female ratio was observed to be $3: 2$ in the present study, the risk of developing aneurysmal subarachnoid hemorrhage for women is 1.6 times than that of men $^{13}$ and most of the authors observed a female predominance ${ }^{9-12}$. The present observation might reflect the health seeking behavior of the study population.

The most common symptoms of SAH namely headache and vomiting ${ }^{13-14}$, was present in all study subjects. Signs of meningeal irritation which was a common sign in $\mathrm{SAH}^{4,14}$ were found in $76.7 \%$ patients in this study. Localizing neurologic signs in patients of SAH include third nerve palsy (posterior communicating aneurysm), sixth nerve palsy (increased intracranial pressure), bilateral lower extremity weakness or abulia (anterior communicating aneurysm), and the combination of hemiparesis and aphasia or visuo-spatial neglect (middle cerebral artery aneurysm $)^{13}$. It has been observed that third cranial nerve palsy was present in $10 \%$ and hemiplegia in $3.3 \%$ of the respondents.

The major identified modifiable risk factors of SAH include smoking, hypertension, cocaine use, and heavy alcohol use ${ }^{13}$. Patients with a family history of first-degree relatives with subarachnoid hemorrhage are also at a higher risk ${ }^{4}$. It was observed in this study that smoking and hypertension was present in almost half of the respondents while only few had family history of SAH. None of the study subjects used alcohol or cocaine, reflecting the religious view of the population.

In this study it was observed that anterior communicating artery was the most common site of aneurysm followed by middle cerebral artery and posterior communicating artery. The findings are 
consistent with those observed by different authors ${ }^{5,-12}$. Most of the patients of this study had medium size aneurysms. However, one third had small size aneurysm that could have been difficult to identify without DSA, as other imaging procedure like CT angiogram often miss them ${ }^{15}$. Morphology of the aneurysms is important in determining subsequent therapeutic procedure. It was found in this study that majority of the aneurysms were saccular and had a narrow neck, which is also reported by most of the observers $^{4,9,14,16}$.

\section{Conclusion}

In conclusion, DSA demonstrated that anterior communicating artery is the most common site of aneurysm in patients with $\mathrm{SAH}$ and are most often saccular with a narrow neck.

Acknowledgements: The authors gratefully acknowledge the scientific colleagues of the department for their generous and moral support to the project. Supporting staffs of the department and patients participating in the project are also thanked and appreciated.

\section{References}

1. Sudlow CL, Warlow CP. Comparable studies of the incidence of stroke and its pathological types: results from an international collaboration. Stroke 1997;28:491-9

2. Johnston SC, Selvin S, Gress DR. The burden, trends, and demographics of mortality from subarachnoid hemorrhage. Neurology 1998;50:1413-8

3. Gijn VJ, Rinkel GJE. Subarachnoid haemorrhage: diagnosis, causes and management. Brain 2001;124:249-278

4. Gijn VJ, Kerr RS, Rinkel GJ. Subarachnoid Hemorrhage: Lancet 2007;369(9558):306-18

5. Peker A, Üstüner E, Özkavukcu E, Sancak T. Performance analysis of 8-channel MDCT angiography in detection, localization, and sizing of intracranial aneurysms identified on DSA. Diagnosis of Interventional Radiology 2009; 15:81-85
6. Randy B, Rocco AA, Patrick N. Modern Imaging in the Management of Cervical Carotid Stenosis. Seminars in Neurosurgery 2002;13(3):1234-38

7. Steffens J, Link JM, Heller MM. Contrast-enhanced magnetic resonance Angiography of the cervical arteries. Investigative Radiology 1998;33:573-77

8. Korogi Y, Takahashi M, Katada K, et al. intracranial aneurysms: detection with three-dimensional $\mathrm{CT}$ angiography with volume rendering-comparison with conventional angiographic and surgical findings. Radiology 1999;211: 497-506

9. Dammert S, Krings T, Moller-Hartmann W, Ueffing E, Hans F.J., K. Willmes K et al. Detection of intracranial aneurysms with multislice CT: comparison with conventional angiography. Neuroradiology 2004;46: 427-434

10. Uysal E, Yanbuloğlu B, Ertürk M, Kılınç BM, Başak M. Spiral $\mathrm{CT}$ angiography in diagnosis of cerebral aneurysms of cases with acute subarachnoid hemorrhage. Diagnosis of Interventional Radiology 2005; 11:77-82

11. Haghighatkhah HR, Sabouri S, Borzouyeh F, Bagherzadeh $\mathrm{MH}$, Bakhshandeh H, Jalali AH. Diagnostic Accuracy of Multi-Slice Computed Tomographic (MSCT) Angiography in the Detection of Cerebral Aneurysms. Iranian Journal of Radiology 2008; 5(4):209-214

12. Colen TW, Wang LC, Ghodke BV, Cohen WA, Hollingworth W, Anzai Y. Effectiveness of MDCT Angiography for the Detection of Intracranial Aneurysms in Patients with Non-traumatic Subarachnoid Hemorrhage. American Journal of Radiology 2007; 189:898-903

13. Suarez JI, Tarr RW, Selman WR. Aneurysmal Subarachnoid Haemorrhage. New England Journal of Medicine 2006;354: 387-96

14. Ropper AH and Samuels MA. Cerebrovascular disease in Adams and Victor's Principles of Neurology. 9th edition. McGraw- Hill; 2009;pp. 808-809

15. Islam $\mathrm{M}$, Chowdhury $\mathrm{AH}$, Chowdhury MSJH, Islam $\mathrm{MN}$, Nayeem A, Khan SU, et al. A comparative study of computed tomographic angiography and digital subtraction angiography in evaluation of aneurysmal subarachnoid hemorrhage. J Dhaka Med Col 2013;22(2):195-200

16. Liang EY, Chan M, Hsiang JHK, Walkden SB, Poon WS, Lam WWM. Metreweli C. Detection and Assessment of Intracranial Aneurysms: Value of CT Angiography with Shaded-Surface Display. American Journal of Radiology 1995;165:1497-1502 